\title{
Stiffness of the Edwards-Anderson Model in all Dimensions
}

\author{
Stefan Boettcher ${ }^{1}$ \\ ${ }^{1}$ Physics Department, Emory University, Atlanta, Georgia 30322, USA
}

\begin{abstract}
A comprehensive description in all dimensions is provided for the scaling exponent $y$ of low-energy excitations in the Ising spin glass introduced by Edwards and Anderson. A combination of extensive numerical as well as theoretical results suggest that its lower critical dimension is exactly $d_{l}=5 / 2$. Such a result would be an essential feature of any complete model of low-temperature spin glass order and imposes a constraint that may help to distinguish between theories.
\end{abstract}

PACS numbers: $05.50 .+\mathrm{q}, 75.10 . \mathrm{Nr}, 02.60 . \mathrm{Pn}$

Imagining physical systems in non-integer dimensions, such as through an $\epsilon$-expansion near the upper [1] or lower critical dimension [2], has provided many important results for the understanding of the physics in realistic dimensions. Often, peculiarities found in unphysical dimensions may impact real-world physics and enhance our understanding $[3$. Here, we will explore the variation in dimension of the low-temperature behavior in the Edwards-Anderson (EA) spin glass model [4].

A quantity of fundamental importance for the modeling of amorphous magnetic materials through spin glasses [5] is the "stiffness" exponent y [6, 7]. As Hooke's law describes the response in increasing elastic energy imparted to a system for increasing displacement $L$ from its equilibrium position, the stiffness of a spin configuration describes the typical rise in magnetic energy $\Delta E$ due to an induced defect-interface of size $L$. But unlike uniform systems with a convex potential energy function over its configuration space (say, a parabola for the sole variable in Hooke's law), an amorphous many-body system exhibits a function more reminiscent of a highdimensional mountain landscape [8]. Any defect-induced displacement of size $L$ in such a complicated energy landscape may move a system through many ups-and-downs in energy $\Delta E$. Averaging over many incarnations of such a system results in a typical energy scale

$$
<|\Delta E|>\sim L^{y} \quad(L \rightarrow \infty) .
$$

The importance of this exponent for small excitations in disordered spin systems has been discussed in many contexts [5, 6, 7, 9, 10, 11, 12.

Spin systems with $y>0$ provide resistance ("stiffness") against the spontaneous formation of defects at sufficiently low temperatures $T$; an indication that a phase transition $T_{c}>0$ to an ordered state exists. For instance, in an Ising ferromagnet, the energy $\Delta E$ is always proportional to the size of the interface, i. e. $y=d-1$, consistent with the fact that $T_{c}>0$ only when $d>1$. When $y \leq 0$, a system is unstable (such as the $d=1$ ferromagnet) to spontaneous fluctuations which proliferate, preventing any ordered state. Thus, determining the "lower critical dimension" $d_{l}$, where $y_{d_{l}}=0$, is of significant importance [6, 13, 14, 15, and understanding the mechanism leading to $d_{l}$, however un-natural, provides definite clues to the origin of order 22. For instance, in homogeneous systems with a continuous symmetry [16], such as the Heisenberg ferromagnet, the possibility of "soft modes" (Goldstone bosons) perpendicular to the direction of magnetization have proved to weaken order further, since defect-interfaces can broaden over the entire length $L$ of the system (spin wave) to reduce the energy by a factor $\sim 1 / L$. This effect manifests itself in a reduced stiffness, $y=d-2$, and an increased $d_{l}=2$.

The addition of quenched disorder drastically complicates the nature of the ordered state [17], since fluctuating interfaces can attain highly irregular geometries to take advantage of heterogeneities. In this case, $y$, and possibly $d_{l}$, may become anomalous. For instance, it was found that adding even a small random field (of zero mean) to an ordinary Ising ferromagnet destabilizes order at least in $d=2$, and considerable insight into the ordered state was gained in proving that $d_{l}=2[18,19,20]$.

For spin glasses, even a numerical determination of $y$ is highly nontrivial when $d>2$ [21], requiring repeated solution of some of the hardest-known combinatorial optimization problems 222]. Thus, simulations have been rather limited in system size $L$ and yielded widely varying results with non-overlapping errors, even for $d=323$. The consensus from these studies is that $2 \leq d_{l} \leq 3 \quad 24$, where $y_{d}$ may behave in a discontinuous manner [25], such that $d_{l}$ could be integer as is the case for other statistical models. On the other extreme, above the "upper critical dimension," well-known to be $d_{u}=6$ 5], where fluctuations become irrelevant, numerical results can be compared with analytical predictions for critical exponents from mean-field theory [26].

In this Letter, we consider an analytic continuation of $y=y_{d}$ to real dimensions $d \geq 0$, based on extensive numerical simulations for $y_{d}$ in integer dimensions 23,27 . That data suggest that $y_{d}$ is varying smoothly across dimensions and that a straightforward low-order fit would provide a very accurate description. The validity and accuracy of this fit is gauged by how well it extrapolates to an exactly known feature, e. g. $y_{1}=-1$. The fit has a zero at $d_{l} \approx 2.4986$ and yields $y_{5 / 2} \approx 0.0008$; strong evidence that $d_{l}=5 / 2$. This value can be corroborated 
by a similar but independent fit of the existing data for $T_{g}$ [28, 29, 30]. A speculative calculation based on meanfield arguments 31], recently put on a more rigorous basis 32], has predicted the disappearance of replica symmetry breaking (RSB) 33], taken to be a characteristic of low- $T$ glassy order, for dimensions $d<d_{l}=5 / 2$ exactly. Our results here lend significant support to the low- $T$ structure of spin glasses advanced in Refs. 31, 32.

Applying a new optimization heuristic 34] to spin glasses on bond-diluted lattices [23] with up to $10^{6}$ spins allowed us to present values for $y_{d}$ in $d=3, \ldots, 7$ that are improved or are entirely unprecedented: $y_{3}=0.24(1)$, $y_{4}=0.61(1), y_{5}=0.88(5), y_{6}=1.1(1)$, and $y_{7}=1.24(5)$. The precision of these values originates from extensive numerical simulations 27] over a large number of systems sizes and bond fractions, yielding 30 or more data points significant for scaling in each dimension, with each point resulting from an average over $10^{4}-10^{6}$ instances.

We can supplement this list of values for $y_{d}$ by those for $d=1$ and 2, assuming a bond distribution that in addition to being symmetric and of unit width also has to be continuous at the origin. Only for $y_{d}>0$ is its value independent of details of the bond distribution 11], where the energy scale in Eq. (11) diverges. For $d \leq 2$, discrete $\pm J$ bonds appear to lead to trivial scaling, $y_{d \leq 2} \equiv 0$ [24, 25], whereas continuous bonds generally provide nontrivial results [24]. For instance, in a $d=1$ spin chain of length $L$, any defect at $T=0$ affects only the absolute weakest bond. Hence, for $\pm J$ bonds, or any other distribution bounded away from the origin, the absolute weakest bond is a non-zero constant for large $L$. For a distribution continuous at the origin, the absolute smallest of $L$ bonds scales as $\sim 1 / L$, thus $y_{1}=-1$ exactly. The value for $y_{2}$ has been considered repeatedly over the years $6,6,35,36,37$, and has been determined with some consistency to be $y_{2}=-0.282(2)$ for continuous bonds. The accuracy for $y_{2}$ is expected to be significantly better than for any $y_{d}$ with $d>2$, since finding exact ground states for $d=2$ spin glasses is accomplished with polynomial time algorithms, facilitating larger sizes and better statistics.

From these values, a clear picture for the stiffness exponent emerges. As Fig. 11 shows, the exponent appears to vary smoothly with dimension $d$, at least between $1 \leq d \leq 6$. (It can be expected that $y_{d}$ would develop a cusp at the upper critical dimension $d_{u}=6$.) Hence, we expect that a simple polynomial fit of the numerical data may provide an accurate interpolation for $y_{d}$. In light of the much reduced uncertainty in the value of $y_{3}$ compared to earlier results, which ranged between $0.19(1)$ to 0.27 23], such a fit should provide an accurate prediction for $d_{l}$, i. e. the zero of $y_{d}$. To fit the data, we proceed in the following way: We fit only the numerical data for $2 \leq d \leq 6$, weighted by their error bars; the quality of the fit is judged on the basis of how well the exactly known data point $y_{1}=-1$ is reproduced. We find that the opti-

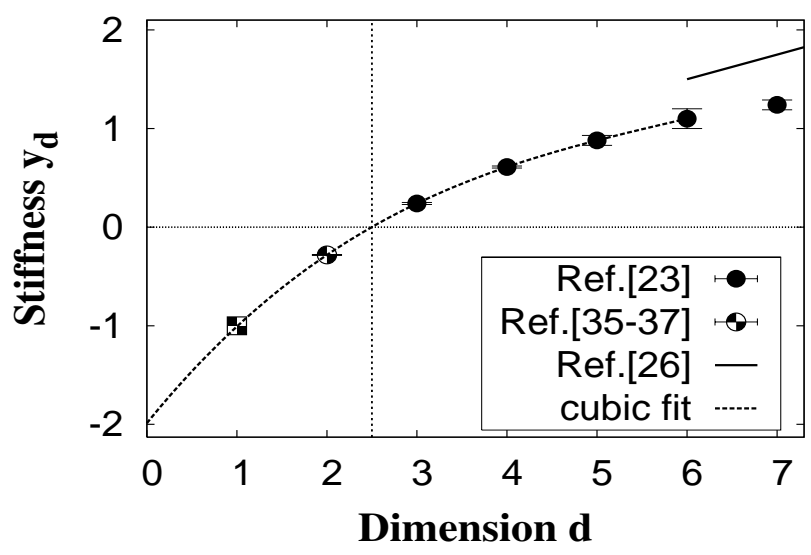

FIG. 1: Plot of the spin glass stiffness exponent $y_{d}$ as a function of dimension $d$. Shown are the data for hypercubic lattices from Refs. 23, 35], a weighted cubic fit to the $y_{d}$ data on $2 \leq d \leq 6$, and the RSB prediction from Ref. [26] in Eq. [6]. The fit predicts to $0.1 \%$ that the lower critical dimension defined through $y_{d_{l}}=0$ is $d_{l}=5 / 2$ (vertical line). It reproduces the exact result, $y_{1}=-1$ (checkered square), to $0.8 \%$ and suggests $y_{0}=-2$. Above the upper critical dimension, $d_{u}=6$, the data significantly deviates from Eq. (6) (solid line).

mal fit is of cubic order: a square fit does not even have enough variability to match all the included data well, let alone $y_{1}$; fits of higher order become unstable since the number of fitting parameters approaches the number of data points. The resulting cubic fit,

$$
y_{d} \approx-1.988+1.125 d-0.1533 d^{2}+0.0086 d^{3},
$$

has a number of desirable properties. In particular, the coefficients for increasing powers of $d$ rapidly decrease, testifying to the stability of the fit. Consequently, Eq. (2) predicts the exactly-known result, $y_{1}=-1$, to less than $0.8 \%$ and further extrapolates to $y_{0}=-2$ (within $0.7 \%$ ). The fit has a zero at $d_{l} \approx 2.4986$ and yields $y_{5 / 2} \approx 0.0008$; suggesting that $d_{l}=5 / 2$.

In fact, a replica theory calculation on a lattice in arbitrary dimensions 31] yields $d_{l}=5 / 2$ exactly. It uses a Landau-like expansion in the neighborhood of $T_{g}$ for the order parameter function for two coupled replicas. Both replicas are held at an identical equilibrium state on one open boundary, and held at two distinct states on the opposite boundary. While the "defect" thus created leads to a different exponent, say, $a_{d} \not \equiv y_{d}$, their zero's should coincide. The defect free energy $\Delta F$ signals that the distinction between overlaps becomes irrelevant exactly in $d=5 / 2$, where replica symmetry gets reestablished throughout. The ad-hoc free-energy extremization in Ref. 31 has recently been put on a more rigorous basis [32], using the inverse range as an expansion parameter near a saddle point of a lattice spin glass with variable interaction range. It is argued that this $\Delta F$ behaves similar to that in ordinary ordered systems with a continuous symmetry, such as the Heisenberg ferromag- 
net, in which the Goldstone mode undermines order already in $d_{l}=2$ [16]. Yet, in the Ising spin glass order is further weakened by the bond disorder without any ferromagnetic bias, making any contribution to the defect energy sub-additive $(\langle\Delta F\rangle=0$ but $\langle|\Delta F|\rangle>0)$, hence $a_{d}=d-2-\frac{1}{2}$. The replica calculation in Refs. 31, 32 is presently the only realistic theory with an analytic prediction for $d_{l}$. Considering the simplicity of a $d_{l}=5 / 2$, one may wonder how essential the replica argument is.

We can put these predictions further into context by comparing with the Migdal-Kadanoff approximation (MK) 38, 39], which has proved to resemble many features of low-dimensional spin glass quite well [40]. In $\mathrm{MK}$, a $d$-dimensional lattice is represented by a hierarchical graph where each bond of range $s \times L$ between two spins consists recursively of $b$ parallel series of $s$ bonds of range $L$, leading to $6,11,24$

$$
d=1+\frac{\ln b}{\ln s} .
$$

Recent simulations of MK in non-integer $d$ (using $b=3$ and continuous $s$ ) have come surprisingly close to the same result [24],$d_{l} \approx 2.5$. Already Ref. [6] found analytically for MK, within a Gaussian approximation, that

$$
y_{d}^{M K} \approx \frac{d-1}{2}+\frac{\ln \left(1-\frac{2}{\pi}\right)}{2 \ln 2},
$$

which has been improved to a $d \rightarrow \infty$ expansion of $y_{d}$ in Ref. 11. Clearly, Eq. (4) fails at $d=1$, and its linear form misses the essential features of the data in Fig. [1 but it provides a decent estimate for $d=2$ and 3 , and pre$\operatorname{dicts} d_{l}^{M K} \approx 2.46$. (Similarly, a suggestion from Ref. 41] that $(d-1) / 2-y=$ const is ruled out by the data.)

Yet, MK predictions are generally ambiguous, since various combinations of $b$ and $s$ in Eq. (3) can represent the same $d$. For instance, for $s \gg 1$ each series of bonds at $T=0$ can be replaced by the (absolute) smallest bond (like the $d=1$ spin chain above). Drawn from a Gaussian distribution of zero mean and unit variance, the equivalent bond replacing each series is of typical size $\sim 1 / s$ and has a random sign. Putting $b$ such iid bonds in parallel thus yields another effective bond of Gaussian distribution with zero mean and width $\sim \sqrt{b} / s$. Hence, for an interfacial energy $\Delta E$ to be scale invariant [i. e. $y=0$ in Eq. (11)] requires $b=s^{2}$ for $b, s \rightarrow \infty$, implying $d_{l}^{M K}=3$ according to Eq. (3). This result is at odds with computations for $b=3$ [24] and variable $s$. Furthermore, using $s=2$ and an analytic continuation for $b \rightarrow 1 / 2$, one can show [42] that $y_{0}^{M K}=-1$; quite inconsistent with Fig. [1]

As an independent test for the picture emerging from Fig. 11 we study the values for the glass-transition temperatures as a function of dimension, $T_{g}(d)$, for $d \geq 3$. To this end, we have gathered the latest values for $T_{g}(d)$ (using $\pm J$ bonds) from the literature, based on simulations [28, 29, 43] or high- $T$ series expansions [30, 44]. We

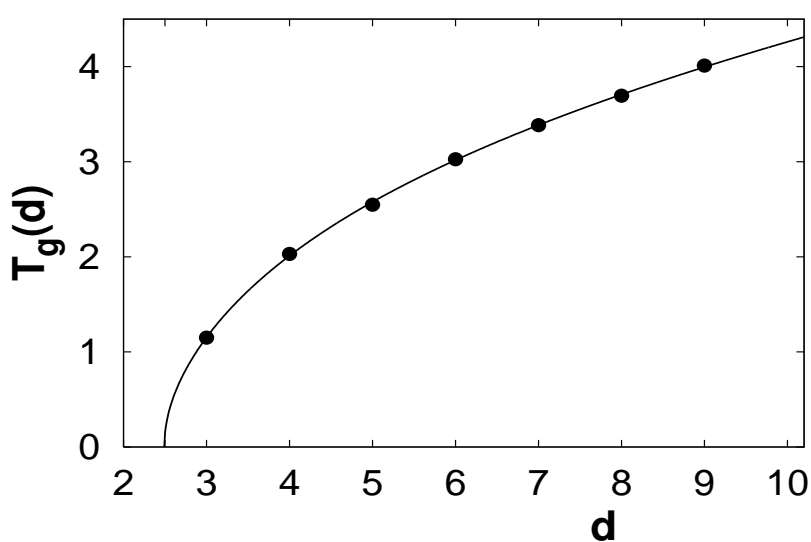

FIG. 2: Plot of the glass transition temperature $T_{g}(d)$ as a function of dimension $d$. Data points are drawn from various studies [28, 29, 30, 43, 44]. The solid line represents the fit in Eq. (5). That fit captures the known asymptotic behavior $T_{g}(d) \sim \sqrt{2 d}$ for $d \rightarrow \infty$ and predicts a zero at $d \approx 2.491$.

found $T_{g}(3) \approx 1.15, T_{g}(4) \approx 2.03, T_{g}(5) \approx 2.548, T_{g}(6) \approx$ $3.026, T_{g}(7) \approx 3.385, T_{g}(8) \approx 3.694$, and $T_{g}(9) \approx 4.011$. Determining $d_{l}$ by fitting $T_{g}(d)$ is somewhat more complicated, since it vanishes for all $d<d_{l}$ (unlike $y_{d}$ ) and Ref. 14] argues that $T_{g}(d) \sim \sqrt{d-d_{l}}$ for $d \rightarrow d_{l}^{+}$. On the other hand, for the Sherrington-Kirkpatrick (SK) model $T_{g}^{S K}=J^{S K}$ 45] and $J \sim J^{S K} / \sqrt{2 d}$, so $T_{g}(d) \sim \sqrt{2 d}$ for $d \rightarrow \infty$ and $J=1$. Satisfying these constraints, we fit

$$
T_{g}(d) \approx \sqrt{2(d-2.491)}\left(1+\frac{1.237}{d}-\frac{2.425}{d^{2}}\right),
$$

which is plotted in Fig. 2 Again, incorporating more terms in the fit leads to drastic oscillations in the coefficients. While the predicted result, $d_{l} \approx 2.491$, is not quite as accurate or stable as above, it is quite consistent. 51]

Unlike the consistency between mean-field arguments and numerics for $d_{l}$, we can observe a large discrepancy for $d \geq d_{u}=6$. An RSB calculations from Ref. [26] around the mean-field limit $(d \rightarrow \infty)$ [4] predicts

$$
y_{d}=d(1-\rho) \approx \frac{d}{4} \quad\left(d \geq d_{u}=6\right),
$$

where the exponent $\rho$, predicted [11, 26] and measured [11, 46, 47] to be $\approx 3 / 4$, describes the width of the distribution of ground state energies in the SK model. As Fig. [1 shows, the finite-dimensional results substantially disagree on this point with replica theory. A plausible interpolation may be $y_{d} \approx(d-2) / 4$, which approaches Eq. (6) for $d \rightarrow \infty$.

In conclusion, we have provided a series of independent, mutually consistent arguments for a lower critical dimension of $d_{l}=5 / 2$ for the Edwards-Anderson spin glass. This self-consistency has a number of important implications. For one, it validates the numerical values for $y_{d}$ for integer dimensions as quoted above [23, 27]; any change 
beyond the error bars of $y_{3}$ in particular would significantly distort the obtained $d_{l}$. The accuracy of those values, in particular $y_{6}$ and $y_{7}$, makes the comparison with the mean-field prediction for $d \geq 6$ relevant, allowing for a rare direct comparison between lattices and mean-field predictions across the dimensions. In turn, the apparent square-root singularity near $d_{l}$ validates the arguments from Ref. 14] for the behavior of $T_{g}$. Most importantly, the consistency of these numerical results with the analytical treatment in Refs. [31, 32] lend credibility to its assumptions and implications. That theory assumes replica symmetry breaking to exist below $T_{g}$ for finite-dimensional lattices, which seems incompatible 48] with the droplet scaling picture developed in Refs. 7, 9]. It further derives that the treatment is unaffected by the existence of an external field, hence predicting an de Almeida-Thouless line, in contrast with some recent experimental [49] and numerical findings [50]. Our findings here suggest a careful reexamination of those ideas.

Many thanks to S. Franz and M. A. Moore for their valuable comments on the manuscript. This work has been supported by grant 0312510 from the Division of Materials Research at the National Science Foundation and by the Emory University Research Council.

[1] K. G. Wilson and M. E. Fisher, Phys. Rev. Lett. 28, 240 (1972).

[2] G. Parisi, Statistical Field Theory (Addison-Wesley, Redwood City, 1988).

[3] C. M. Bender, S. Boettcher, and L. Lipatov, Phys. Rev. Lett. 68, 3674 (1992).

[4] S. F. Edwards and P. W. Anderson, J. Phys. F: Metal Phys. 5, 965 (1975).

[5] K. H. Fischer and J. A. Hertz, Spin Glasses (Cambridge University Press, Cambridge, 1991).

[6] B. W. Southern and A. P. Young, J. Phys. C: Solid State Phys. 10, 2179 (1977).

[7] A. J. Bray and M. A. Moore, J. Phys. C: Solid State Phys. 17, L463 (1984).

[8] H. Frauenfelder, ed., Landscape Paradigms in Physics and Biology (Elsevier, Amsterdam, 1997).

[9] D. S. Fisher and D. A. Huse, Phys. Rev. Lett. 56, 1601 (1986).

[10] M. Palassini and A. P. Young, Phys. Rev. Lett. 85, 3017 (2000).

[11] J.-P. Bouchaud, F. Krzakala, and O. C. Martin, Phys. Rev. B 68, 224404 (2003).

[12] F. Krzakala and O. Martin, Phys. Rev. Lett. 85, 3013 (2000).

[13] W. L. McMillan, Phys. Rev. B 30, 476 (1984).

[14] D. S. Fisher and D. A. Huse, Phys. Rev. B 38, 386 (1988).

[15] J. Maucourt and D. R. Grempel, Phys. Rev. Lett. 80, 770 (1998).

[16] N. Goldenfeld, Lectures on Phase Transitions and the Renormalization Group (Addison-Wesley, Reading, 1992).

[17] Y. Imry and S. k. Ma, Phys. Rev. Lett. 35, 1399 (1975).
[18] D. Andelman and H. O. L. C. R. Wijewardhana, Phys. Rev. Lett. 52, 145 (1984).

[19] J. Z. Imbrie, Phys. Rev. Lett. 53, 1747 (1984).

[20] J. Bricmont and A. Kupiainen, Phys. Rev. Lett. 59, 1829 (1987).

[21] F. Barahona, J. Phys. A: Math. Gen. 15, 3241 (1982).

[22] H. Rieger and A. Hartmann, eds., New Optimization Algorithms in Physics (Springer, Berlin, 2004).

[23] S. Boettcher, Europhys. Lett. 67, 453 (2004).

[24] C. Amoruso, E. Marinari, O. C. Martin, and A. Pagnani, Phys. Rev. Lett. 91, 087201 (2003).

[25] A. K. Hartmann and A. P. Young, Phys. Rev. B 64, 180404(R) (2001).

[26] T. Aspelmeier, M. A. Moore, and A. P. Young, Phys. Rev. Lett. 90, 127202 (2003).

[27] S. Boettcher, Euro. Phys. J. B 38, 83 (2004).

[28] H. G. Ballesteros, A. Cruz, L. A. Fernandez, V. MartaMayor, J. Pech, J. J. Ruiz-Lorenzo, A. Tarancan, P. Tallez, C. L. Ullod, and C. Ungil, Phys. Rev. B 62, 14237 (2000).

[29] L. W. Bernardi and I. A. Campbell, Phys. Rev. B 56, 5271 (1997).

[30] L. Klein, J. Adler, A. Aharony, A. Harris, and Y. Meir, Phys. Rev. B 43, 11249 (1991).

[31] S. Franz, G. Parisi, and M. A. Virasoro, J. Phys. I (France) 4, 1657 (1994).

[32] S. Franz and F. L. Toninelli, J. Stat. Mech. p. P01008 (2005).

[33] M. Mézard, G. Parisi, and M. A. Virasoro, Spin glass theory and beyond (World Scientific, Singapore, 1987).

[34] S. Boettcher and A. G. Percus, Phys. Rev. Lett. 86, 5211 (2001).

[35] H. Rieger, L. Santen, U. Blasum, M. Diehl, and M. Jünger, J. Phys. A: Math. Gen. 29, 3939 (1996).

[36] A. K. Hartmann, A. J. Bray, A. C. Carter, M. A. Moore, and A. P. Young, Phys. Rev. B 66, 224401 (2002).

[37] S. Boettcher and A. K. Hartmann, Phys. Rev. B 72, 014429 (2005).

[38] A. A. Migdal, J. Exp. Theo. Phys. 42, 743 (1976).

[39] L. P. Kadanoff, Ann. Phys. 100, 359 (1976).

[40] S. Boettcher and S. E. Cooke, Phys. Rev. B 71, 214409 (2005).

[41] M. Cieplak and J. R. Banavar, J. Phys. A: Math. Gen. 23, 4385 (1990).

[42] S. Boettcher, unpublished.

[43] E. Marinari, G. Parisi, and J. J. Ruiz-Lorenzo, in Spin Glasses and Random Fields, edited by A. P. Young (World Scientific, Singapore, 1998).

[44] D. Daboul, I. Chang, and A. Aharony, Eur. Phys. J. B 41, 231 (2004).

[45] D. Sherrington and S. Kirkpatrick, Phys. Rev. Lett. 35, 1792 (1975).

[46] M. Palassini, cond-mat/0307713.

[47] S. Boettcher, Eur. Phys. J. B 46, 501 (2005).

[48] C. M. Newman and D. L. Stein, Phys. Rev. Lett. 76, 515518 (1996).

[49] P. E. Jönsson, H. Takayama, H. Aruga Katori, and A. Ito, Phys. Rev. B 71, 180412(R) (2005).

[50] A. P. Young and H. G. Katzgraber, Phys. Rev. Lett. 93, 207203 (2004).

[51] A similar fit has been attempted already in G. Parisi, P. Ranieri, F. Ricci-Tersenghi, and J. J. Ruiz-Lorenzo, J. Phys. A: Math. Gen. 30, 7115 (1997). 